Departamento de Ciência e Tecnologia, Secretaria de Ciência, Tecnologia e Insumos Estratégicos, Ministério da Saúde

Correspondência | Correspondence:

Decit - Departamento de Ciência e Tecnologia

do Ministério da Saúde

Esplanada dos Ministérios Bloco G sala 845

70058-900 Brasília, DF, Brasil

Texto de difusão técnico-científica do

Ministério de Saúde

\section{Programa de Desenvolvimento Institucional do Sistema Único de Saúde}

\section{Institutional Development Program of the Brazilian National Health System}

O Programa de Desenvolvimento Institucional do Sistema Único de Saúde (Proadi-SUS) é uma ação do Ministério da Saúde (MS) dirigida ao fortalecimento do SUS em parceria com hospitais filantrópicos de qualidade reconhecida. Essa parceria reflete o comprometimento com a melhoria da qualidade das condições de saúde da população brasileira mediante transferência, desenvolvimento e incorporação de novos conhecimentos e práticas em áreas estratégicas para o sistema. Baseadas em temas prioritários determinados pelo MS, as instituições parceiras apresentam projetos a serem executados em um período de três anos nas seguintes áreas: estudos de avaliação e incorporação de tecnologia, capacitação de recursos humanos, pesquisas de interesse público em saúde e desenvolvimento de técnicas e operação de gestão em serviços de saúde. O programa segue a orientação constitucional apoiada no tripé da universalidade, integralidade e eqüidade do SUS.

O Proadi foi instituído pelo Decreto $\mathrm{n}^{\circ} 2.536$, de 7 de abril de 1998, que dispunha sobre a concessão do Certificado de Entidade de Fins Filantrópicos a que se refere o inciso IV do art. 18 da Lei $n^{\circ} 8.742$, de 7 de dezembro de 1993, e dá outras providências. Esse decreto determinou pela primeira vez a possibilidade de as entidades de filantropia se beneficiarem de isenções fiscais por meio de projetos voltados ao SUS. De acordo com o parágrafo 17 do artigo 3 do Decreto $\mathrm{n}^{\circ} 2.536$, adicionado pelo Decreto ${ }^{\circ} 5.895$, de 8 de agosto de 2006, "A instituição de saúde poderá, alternativamente, para dar cumprimento ao requisito previsto no inciso VI do caput deste artigo ou no $\S 4^{\circ}$, realizar projetos de apoio ao desenvolvimento institucional do SUS, estabelecendo convênio com a União, por intermédio do Ministério da Saúde". A regulamentação para o início das atividades de apoio ao SUS pelas entidades filantrópicas foi instituída pela Portaria $\mathrm{n}^{\circ} 3.276$, de 28 de dezembro de 2007, a qual estabelece que as instituições que optarem por desenvolver projetos de apoio ao desenvolvimento institucional do SUS deverão atender as etapas de habilitação e a apresentação de projetos.
Posteriormente, foi publicada a Lei $\mathrm{n}^{\circ} 12.101$, de 27 de novembro de 2009, que dispõe sobre a certificação das entidades beneficentes de assistência social e regula os procedimentos de isenção de contribuições sociais. De acordo com o artigo 11 dessa lei, para ser considerada beneficente e fazer jus à certificação, a entidade de saúde de reconhecida excelência poderá realizar projetos de apoio ao desenvolvimento institucional do SUS, celebrando ajuste com a União, por intermédio do MS, em qualquer das áreas de interesse do SUS. Essa lei estabelece, como critério alternativo para as instituições filantrópicas habilitadas como de excelência, a possibilidade de substituição da obrigatoriedade de $60 \%$ da sua atividade assistencial prestada ao SUS e remunerada segundo tabela SUS, pela opção de se integrar ao ProadiSUS, apresentando projetos cuja somatória financeira não deverá ser inferior ao valor total da isenção fiscal decorrente da certificação de filantropia.

A Lei no 12.101 atribui o termo "excelência" para as instituições que participam dos benefícios de isenção. Estabeleceu-se, assim, a expressão "Hospitais de Excelência" (HE), nome hoje utilizado por essas instituições para reconhecer que foram habilitadas pelo MS e cumprem os requisitos para a apresentação de projetos de apoio ao SUS em troca de isenção de contribuições sociais. A denominação indica que as instituições de saúde assim reconhecidas passam a integrar as ações estratégicas do SUS de maneira mais intensa por conta do uso otimizado de sua capacidade técnica e conhecimento. Seis hospitais obtiveram o reconhecimento como HE, sendo cinco da cidade de São Paulo:

- Hospital Alemão Oswaldo Cruz (HAOC - SP)

- Hospital do Coração (HCor - SP)

- Hospital Israelita Albert Einstein (HIAE - SP)

- Hospital Moinhos de Vento (HMV - RS)

- Hospital Samaritano (SP)

- Hospital Sírio Libanês (HSL - SP) 
Os termos de ajuste com os HE foram firmados em novembro de 2008. Desde então essas instituições começaram a apresentar projetos nas quatro áreas visando melhoria dos serviços do SUS e iniciando-se uma nova etapa da filantropia no País. Os projetos de apoio ao desenvolvimento institucional do SUS devem ser apresentados pelos HE ao MS, que os analisa, e, uma vez aprovados, são desenvolvidos com financiamento dos próprios hospitais até o valor máximo da isenção tributária a que têm direito em função do Certificado de Entidade Beneficente de Assistência Social em Saúde (Cebas).

Considerando a nova legislação para a filantropia, Lei ${ }^{\circ}$ $12.101 / 2009$ e Dec. No $7237 / 2010$, houve a necessidade de se estabelecer, no âmbito do SUS, ato normativo para se adequar ao novo regramento e também definir critérios de monitoramento e avaliação eficientes de modo a fortalecer o Proadi-SUS. Em 27 de abril de 2011 foi publicada a Portaria GM/MS n ${ }^{\circ}$ 936, que dispõe sobre o Proadi, destacando a criação de comitê gestor, formado por representantes de todas as unidades do MS, Agência Nacional de Vigilância Sanitária, Agência Nacional de Saúde, Conselho Nacional de Secretários de Saúde e Conselho Nacional de Secretarias Municipais de Saúde, ao qual competem:

- definição dos temas e objetivos prioritários;

- definição de critérios e requisitos para a comprovação de efetiva capacidade institucional da entidade de saúde;

- aprovação da carta consulta;

- avaliação dos resultados dos projetos de apoio; e

- formulação de proposições para aprimoramento do Proadi.

Atualmente, estão em acompanhamento 112 projetos do Proadi aprovados no primeiro triênio. A maioria deles trata de novas modalidades de atenção e uso de tecnologias avançadas na gestão de estabelecimentos de saúde e no atendimento a pacientes encaminhados pelas instituições pertencentes ao SUS até os HE.

O Departamento de Ciência e Tecnologia (Decit) da Secretaria de Ciência, Tecnologia e Insumos Estratégicos (SCTIE) do MS recebeu para análise e posterior acompanhamento 40 projetos: 26 avaliados pela Coordenação-Geral de Pesquisa Clínica (CGPC), 13 pela Coordenação-Geral de Avaliação de Tecnologias em Saúde (CGATS) e um pela Coordenação-Geral de Fomento à Pesquisa em Saúde (CGFPS). Dos 40 projetos analisados, 32 foram aprovados para execução no triênio 2009-2011 e relacionados na Tabela.

\section{PESQUISA CLÍNICA NO ÂMBITO DO PROADI}

A maioria dos projetos de pesquisa clínica corresponde a pesquisas de caráter epidemiológico e ensaios clínicos. Três projetos de capacitação de recursos humanos foram direcionados para os integrantes da Rede Nacional de Pesquisa Clínica (RNPC), os quais vêm se beneficiando desses treinamentos oferecidos pelos HE, sendo dois na modalidade presencial na cidade de São Paulo e um misto (presencial e a distância). Os recursos disponibilizados para os projetos de pesquisa e de treinamento em pesquisa clínica foram de aproximadamente R\$ 17 milhões, correspondendo a um investimento médio de quase $\mathrm{R} \$ 1$ milhão por projeto.

\section{AVALIAÇÃO DE TECNOLOGIAS EM SAÚDE NO ÂMBITO DO PROADI}

Dentre os projetos relacionados à avaliação de tecnologias em saúde, quatro são destinados à consolidação de unidades de suporte à tomada de decisão, envolvendo tecnologias biomédicas, no apoio à formação de recursos humanos e no suporte a ações institucionais. Esses projetos, em consonância com outras iniciativas do Decit, favorecem o fortalecimento da avaliação de tecnologias em saúde no SUS, mediante produção de pareceres técnico-científicos, elaboração de diretriz metodológica, fomento à educação continuada (envolvendo mais de sete mil alunos) e suporte aos eixos estratégicos da Rede Brasileira de Avaliação de Tecnologias em Saúde. ${ }^{a}$ Destaca-se, ainda, que dois HE estão envolvidos na organização da $8^{\mathrm{a}}$ edição do encontro da Sociedade Health Technology Assessment International (HTAi) na cidade do Rio de Janeiro. Outros projetos para o desenvolvimento de tecnologia que envolve procedimentos robóticos não disponíveis no País também têm sido fomentados, contribuindo para o desenvolvimento de tecnologias emergentes não custeadas pelo SUS. Como exemplo, estão os estudos relacionados à cardiopatia congênita, à insuficiência cardíaca e ao monitoramento do horizonte tecnológico.

O Proadi-SUS permite a construção de conhecimentos e práticas úteis ao sistema de saúde, desenvolvidos em uma parceria público-privada, i.e., entre o Estado e a sociedade. Essa parceria sugere que os valores de isenção de impostos dos HE possam ser convertidos de forma positiva quando aplicados à pesquisa e ao treinamento de recursos humanos. Ressalta-se que os projetos em acompanhamento são auditados quanto à aplicação adequada de recursos por auditores externos independentes.

Como exemplo: o estudo ACT (ou ACT Trial) teve como objetivo avaliar a eficácia de um fármaco (nacetilcisteína), utilizado para prevenir a ocorrência de nefropatia induzida por contraste em pacientes de risco (nefropatas, diabéticos, idosos). Esse ensaio clínico

\footnotetext{
a Mnistério da Saúde. Brasileira de Avaliação de Tecnologias em Saúde. [página na internet] Disponível em: www.saude.gov.br/rebrats
} 
envolveu 46 centros brasileiros e foi coordenado pelo Instituto de Pesquisas do Hospital do Coração de São Paulo. Foram recrutados 2.308 pacientes e os resultados mostraram que não houve efeito protetor do medicamento utilizado. As conclusões do estudo foram apresentadas no Congresso da American Heart Association.

Os resultados desse estudo e dos demais, a serem concluídos até o final de 2011, devem ser utilizados para orientar a tomada de decisões de gestores, assim como de profissionais da saúde nas especialidades contempladas pelos estudos, como cardiologia, oncologia, nefrologia pediátrica e neurologia.

\section{COMENTÁRIOS FINAIS}

A experiência do primeiro triênio de execução dos projetos do Proadi indica que a análise minuciosa das propostas apresentadas, aliada ao acompanhamento criterioso dos projetos selecionados nas áreas de Pesquisa Clínica e Avaliação de Tecnologias em Saúde, pode contribuir com resultados importantes para o SUS.

O Ministério da Saúde, por meio da Secretaria Executiva, vem aprimorando as tarefas de controle e acompanhamento desses projetos. Recentemente, foram publicadas a Portaria $\mathrm{n}^{\circ} 233$, de 16 de março de 2011, que definiu as áreas temáticas e os objetivos prioritários para a elaboração de projetos de apoio ao desenvolvimento institucional do SUS, e a Portaria $\mathrm{n}^{\circ}$ 936, de 27 de abril de 2011, que estabelece as regras e critérios para apresentação, monitoramento, acompanhamento e avaliação de projetos do Proadi-SUS para o segundo triênio.

Quadro. Projetos de pesquisa e apoio ao desenvolvimento institucional do SUS em acompanhamento pelo Departamento de Ciência e Tecnologia da Secretaria de Ciência, Tecnologia e Insumos Estratégicos do Ministério da Saúde, 2011.

\begin{tabular}{|c|c|}
\hline Projeto & Hospital \\
\hline \multicolumn{2}{|l|}{ Coordenação Geral de Pesquisa Clínica } \\
\hline $\begin{array}{l}\text { Estudo de coorte retrospectivo sobre a mortalidade por câncer de mama estágio } 2 \mathrm{~B} \text { e } \\
\text { câncer de pulmão estágio } 3 \mathrm{~A} \text {, comparando hospitais privados e hospitais do SUS }\end{array}$ & \multirow{2}{*}{$\begin{array}{l}\text { Hospital Alemão } \\
\text { Oswaldo Cruz - HAOC }\end{array}$} \\
\hline Programa Nacional de Capacitação em Pesquisa Clínica & \\
\hline \multicolumn{2}{|l|}{$\begin{array}{l}\text { Comparação entre o uso de anticoagulante oral e o antiagregante plaquetário na profilaxia do } \\
\text { tromboembolismo no pós-operatório da operação cavo-pulmonar total: estudo randomizado }\end{array}$} \\
\hline \multicolumn{2}{|l|}{ Estudo ACT (Acetilcisteína para prevenção de nefropatia induzida por contraste) } \\
\hline \multicolumn{2}{|l|}{$\begin{array}{l}\text { Estudo PillPilot - Organização Mundial da Saúde (avaliação de uma polipílula de baixo } \\
\text { custo para pacientes com risco cardiovascular intermediário) }\end{array}$} \\
\hline \multicolumn{2}{|l|}{ Estudo Vision - Vascular events in noncardiac surgery patients cohort evaluation study } \\
\hline $\begin{array}{l}\text { A eficácia de reposição de nicotina e terapia cognitivo-comportamental em grupo versus } \\
\text { reposição de nicotina e o cuidado usual na cessação do tabagismo e prevenção de recaída: } \\
\text { um estudo controlado e randomizado }\end{array}$ & $\begin{array}{l}\text { Hospital do Coração - } \\
\text { HCor }\end{array}$ \\
\hline \multicolumn{2}{|l|}{$\begin{array}{l}\text { Space (anteriormente denominado Umpire) - Single Pill to Avert Cardiovascular Events - } \\
\text { Efeito de polipílula em pacientes de alto risco cardiovascular: um ensaio clínico randomizado }\end{array}$} \\
\hline \multicolumn{2}{|l|}{$\begin{array}{l}\text { Validação de um programa de modificação de estilo de vida (dieta cardioprotetora } \\
\text { brasileira + orientação de atividade física) de baixo custo }\end{array}$} \\
\hline \multicolumn{2}{|l|}{ Programa de formação em pesquisa clínica } \\
\hline \multicolumn{2}{|l|}{$\begin{array}{l}\text { Avaliação do acúmulo do ferro hepático, esplênico cardíaco e pancreático em portadores } \\
\text { de Talassemia Major }\end{array}$} \\
\hline $\begin{array}{l}\text { Avaliação da incidência clínica e prognóstica das mutações em pacientes com leucemia } \\
\text { mielóide aguda de novo e comparação de dois esquemas de consolidação }\end{array}$ & $\begin{array}{l}\text { Hospital Israelita Albert } \\
\text { Einstein - HIAE }\end{array}$ \\
\hline \multicolumn{2}{|l|}{$\begin{array}{l}\text { Monitorização sérica dos níveis de bussulfano em pacientes submetidos a transplante de } \\
\text { medula óssea }\end{array}$} \\
\hline \multicolumn{2}{|l|}{$\begin{array}{l}\text { Projeto de pesquisa em nefrologia: prevalência da doença renal crônica em crianças no } \\
\text { Estado de São Paulo }\end{array}$} \\
\hline \multicolumn{2}{|l|}{$\begin{array}{l}\text { Projeto de pesquisa em nefrologia: indicação e benefícios da utilização da técnica de } \\
\text { hemodiálise diária em crianças com doença renal crônica }\end{array}$} \\
\hline $\begin{array}{l}\text { Desenvolvimento do núcleo de pesquisa e aplicação de técnicas avançadas em neurocirurgia } \\
\text { funcional: implantação de projeto de cirurgia funcional em Doença de Parkinson e } \\
\text { elucidação dos mecanismos de controle neural dos movimentos (Projeto Neurociência) }\end{array}$ & Hospital Sírio Libanês \\
\hline $\begin{array}{l}\text { Uso da cirurgia robótica no tratamento de carcinomas epidermóides em Cirurgia de Cabeça } \\
\text { e Pescoço: Tumores de faringe como instrumento para a melhoria de qualidade de vida }\end{array}$ & $-\mathrm{HSL}$ \\
\hline Treinamento prático em pesquisa clínica e capacitação dos CEPs em hospitais do SUS & \\
\hline
\end{tabular}


Tabela continuação

\begin{tabular}{lc}
\hline Projeto & Hospital \\
\hline Coordenação Geral de Avaliação de Tecnologias em Saúde & \\
Avaliação da viabilidade econômica da oclusão percutânea da comunicação interatrial do & \\
tipo ostium secundum em crianças e adolescentes & \\
Avaliação da viabilidade econômica da oclusão percutânea da persistência do canal arte- \\
rial em crianças e adolescentes \\
Avaliação da viabilidade, segurança e eficácia da oclusão percutânea e perventricular da \\
comunicação interventricular muscular em lactentes, crianças e adolescentes \\
Bomba de Sangue Centrífuga Implantável para Assistência Uni e Biventricular & Hospital do Coração \\
Centro de Medicina Baseada em Evidências para suporte na elaboração de Pareceres & Hospital Alemão \\
Técnico-científicos para o MS & Oswaldo Cruz - HAOC \\
Centro Revisões Sistemáticas e Metanálises & Hospital Sírio-Libanês \\
Desenvolvimento de Modelo de Coração Artificial Auxiliar & - HSL \\
Projeto de apoio à Rede Brasileira de Avaliação de Tecnologias em Saúde (Rebrats) & Hospital Israelita Albert \\
Projeto de revisão sistemática da literatura nas cirurgias assistidas por robô & Einstein - HIEA \\
Capacitação em saúde baseada em evidências & Hospital Samaritano \\
Diagnóstico molecular em tumores hereditários - BRCA & Hírio-Libanês \\
Radioterapia estereotática corpórea hipofracionada no tratamento de neoplasias de pulmão \\
estádios I e II clinicamente inoperáveis \\
Indicadores de Avaliação da Qualidade dos Programas de Controle de Infecção Hospitalar \\
Coordenação Geral de Fomento à Pesquisa em Saúde \\
Atividade antitumoral de extratos de plantas brasileiras. Um projeto de bioprospecção na \\
bacia do Rio Negro, Amazônia
\end{tabular}

